\title{
Extracción de Antioxidantes de las Bayas del Sauco (Sambucus nigra L. subsp. peruviana) con Ultrasonido, Microondas, Enzimas y Maceración para la obtención de Zumos Funcionales
}

\author{
Edilberto Flores \\ Universidad Católica de Santa María, Facultad de Ciencias e Ingenierías Biológicas y Químicas, Escuela \\ Profesional de Ingeniería de Industria Alimentaria, Urb. San José s/n Umacollo, Arequipa - Peru \\ (email: edicato@yahoo.es)
}

Recibido May. 26, 2016; Aceptado Jul. 22, 2016; Versión final Sep. 13, 2016, Publicado Feb. 2017

\begin{abstract}
Resumen
El objetivo de esta investigación fue evaluar el uso de enzimas, ultrasonido, microondas y maceración para mejorar la extracción de antioxidantes de las bayas de sauco. El estudio se justifica por el gran interés por parte de los consumidores y sector empresarial en bebidas que contienen antioxidantes; El contenido de antocianinas se determinó por el método diferencial de $\mathrm{pH}$, fenoles totales por el método de Folin, la capacidad antioxidante por la reducción del ion cúprico y captura del radical catión del ácido 2,2'-azinobis- (3-etillbenzotiazolin-6-sulfonico). La maceración a $70{ }^{\circ} \mathrm{C}$ por 20 minutos origino mejores características antioxidantes, seguida del uso de microondas, enzimas y ultrasonido. La combinación de enzimas-microondas mejora el proceso de extracción, dependiendo del tipo de enzima. El empleo de enzimas permitió mayores rendimientos de extracción en volumen de zumo. Con el zumo obtenido por maceración se elaboró una bebida de sauco con buenas características antioxidantes y sensoriales.
\end{abstract}

Palabras clave: antocianinas; zumos antioxidantes; extracción de compuestos antioxidantes; capacidad antioxidante; frutas rojas.

\section{Antioxidant Extraction from Elderberries (Sambucus nigra $L$. Subsp. peruviana) with Ultrasound, Microwave, Enzymes, and Maceration to obtain Functional Juices}

\begin{abstract}
The objective of this research was to evaluate the use of enzyme-assisted, ultrasound, microwave-assisted and maceration in order to improve its yield of extraction from elderberries. The study is justified because of the great interest by consumers and manufactures on drinks that contain antioxidants. The anthocyanin content, total phenols and antioxidant capacity were determined by the differential method of $\mathrm{pH}$, Folin method and by reducing the cupric ion and radical capture cation-2,2'-azino bis (3-etillbenzotiazolin -6sulfonic acid), respectively. Maceration at $70^{\circ} \mathrm{C}$ for 20 minutes generated the product with the best antioxidant properties when compared to those generated by microwave-assisted extraction, enzymesassisted and ultrasound methods in that order. The combination of enzymes-microwave improves the extraction process, depending on the type of enzyme.The use of enzymes allowed higher extraction yields of extracted juice. With the extracted juice, a beverage with high content of antioxidants and good sensory characteristics was prepared.
\end{abstract}

Keywords: anthocyanins; antioxidant juices, extraction of antioxidants; antioxidant capacity; berries 


\section{INTRODUCCION}

Las bayas constituyen entre otros, un tipo de alimentos más ricas de antioxidantes: vitamina $\mathrm{C}$ y flavonoides que dan su color y pueden ayudar a reducir el riesgo de enfermedades del corazón, cáncer y pérdida de memoria. Los flavonoides protegen al organismo del daño producido por agentes oxidantes como rayos UV, contaminación ambiental, sustancias químicas presentes en los alimentos, estrés y otros, poseen actividad antioxidante, antiinflamatoria, antitumoral e inhibidora de la acción plaquetaria. El organismo humano no produce dichas sustancias debiendo ser obtenidas por la alimentación. (Vilaplana, 2005; Coronado, et al., 2015). El sauco (Sambucus nigra L. Subsp. Peruviana) y sus respectivos zumos tienen efecto antioxidante, antiviral, antihipertensivo y otros efectos favorables debido a componentes fenólicos (incluyendo las antocianinas), terpenoides y proteínas (lecitinas) entre otros, también muestra efecto diurético y alivian el estreñimiento, además ofrecen protección contra el cáncer a la próstata y enfermedades neurodegenerativas (Smith et al., 2014).

Las antocianinas pertenecen al grupo de los flavonoides, son un grupo de pigmentos con estructuras fenólicas variadas siendo poderosos antioxidantes (Volp et al., 2008). La presencia de flavonoides y antocianinas en las bayas son valoradas por sus propiedades beneficiosa a la salud (Jakobek et al., 2007, García et al., 2002). La estabilidad del color de las antocianinas es dependiente de la estructura y de la concentración de los pigmentos, además de factores como el pH, influencia de la copigmentación, luz, temperatura, presencia de metales, oxigeno (López et al., 2007, Damodaran et al., 2010).

Además de los procesos tradicionales como la maceración (Ćujić et al., 2016), nuevas tecnologías vienen siendo aplicadas para mejorar los procesos de extracción como la homogeneización ultrasónica (Carrera et al., 2012), que permite alcanzar una reducción de partículas blandas y duras por intensas fuerzas de cavitación, que acelera los procesos de extracción y mejoran los procesos de transferencia de masas. El uso de enzimas (Maier et al., 2007) mejoran la extracción de antocianinas en la cáscara de uva toda vez que el propósito principal del uso de enzimas es alterar la estructura de la pared celular y mejorar el rendimiento de extracción del jugo y como consecuencia liberar componentes fenólicos al jugo entre otros. Microondas fueron también aplicadas en procesos de extracción de antocianinas en hollejos de uva (Liazid et al., 2011), las ondas electromagnéticas interaccionan con las moléculas de agua en el alimento las cuales vibran o rotan por efecto de las microondas lo cual genera calor y que pueden favorecer los procesos de difusión de diversos componentes a la fase liquida. Diversos trabajos evalúan el empleo de solventes orgánicos como el etanol en los procesos de extracción empleando estas tecnologías. Los procesos de extracción acuosa de sustancias bioactivas cobran importancia en la elaboración de productos no alcohólicos como el caso de las bebidas funcionales.

El proceso de elaboración de jugos de bayas incluye una etapa de molienda, un tratamiento enzimático y prensado para obtener un jugo crudo y una parte sólida (Ávila de Hernández y Bullón, 2013). En la elaboración de jugos funcionales interesa extraer y preservar la mayor cantidad de componentes bioactivos beneficiosas a la salud en los productos finales. Las características funcionales de las bebidas elaboradas provenientes de bayas dependerán del modo de su elaboración, de los niveles de extracción de los componentes bioactivos de las materias primas empleadas, de los procesos térmicos aplicados, envasado, condiciones de almacenamiento. Las temperaturas de almacenamiento influyen en la estabilidad de las antocianinas en las bebidas, son favorables temperaturas de $4{ }^{\circ} \mathrm{C}$ frente a las de $25^{\circ} \mathrm{C}$ (Arrasola et al., 2013). Se espera que las tecnologías emergentes aplicadas a las bayas de saúco favorezcan la extracción de sustancias antioxidantes en el proceso de elaboración de zumos.

El objetivo de esta investigación fue evaluar la extracción de sustancias antioxidantes de las bayas del sauco con diferentes técnicas de extracción (enzimas, ultrasonido, microondas o sus combinaciones, maceración), con la finalidad de obtener zumos de sauco con alto contenido de antocianinas, fenoles totales y capacidad antioxidante. Las diversas extracciones se efectuaron en el mismo jugo de la baya de sauco en contacto con la cascara. Con la metodología de extracción más favorable se elaboró una bebida rica en antioxidantes.

\section{MATERIALES Y METODOS}

La metodología se presenta en cinco subsecciones, en una se señala la procedencia de las bayas de sauco, su almacenamiento y empleo, luego se describen los diversos procedimientos de extracción empleados, haciendo referencia al zumo control, en una tercera parte se explica el procedimiento de elaboración de la bebida, en una cuarta parte se indican los métodos de análisis empleados para evaluar las características antioxidantes de los jugos extraídos por los diversos tratamientos aplicados y los análisis físico químicos realizados en el zumo control y en la bebida elaborada, y finalmente se presenta el análisis estadístico empleado. 


\section{Procedencia de las Bayas de sauco y tratamiento}

Las bayas de sauco fueron procedentes del Centro Experimental Andenes ubicada en el Departamento del Cuzco- Perú, después de recolectadas se congelaron a $-12{ }^{\circ} \mathrm{C}$ en bolsas de polietileno conteniendo $200 \mathrm{~g}$ cada una. Para su uso en los diversos tratamientos las bayas se descongelaron hasta alcanzar una temperatura de $20^{\circ} \mathrm{C}$ en un tiempo aproximado de 10 minutos. Se colocó $200 \mathrm{~g}$ de bayas descongeladas en un vaso de $1000 \mathrm{ml}$ y se desmenuzaron con un procesador manual de vegetales marca Philips HR 1357, $300 \mathrm{~W}$.

El sauco triturado se empleó en los diversos tratamientos de extracción evaluados: maceración, ultrasonido, enzimas, microondas o tratamientos combinados; para tal fin se colocaron $100 \mathrm{~g}$ de sauco en vasos de 250 $\mathrm{ml}$; después de aplicado el tratamiento de extracción se repone el agua si es que hubo evaporación, luego se prensa a través de un cedazo y se mide el volumen extraído y las características antioxidantes de los zumos obtenidos después de los tratamientos efectuados: contenido de antocianinas, fenoles totales y capacidad antioxidante.

\section{Procedimientos de extracción y zumo control}

La maceración del jugo de las bayas trituradas junto con las cascaras y sin adición de agua se efectúo a 30 , $50,70{ }^{\circ} \mathrm{C}$ por 10 minutos. Con la mejor temperatura de extracción de antioxidantes se realizó extracciones por 5, 10, 20 y $30 \mathrm{~min}$. Adicionalmente se efectuaron pruebas de extracción a $80^{\circ} \mathrm{C}$ por $5,10,20$ y $30 \mathrm{~min}$. El zumo control se obtuvo por prensado simple de las bayas de sauco trituradas en el procesador, en la cual se determinó el volumen obtenido y las características antioxidantes y físico químicas.

El tratamiento enzimático se realizó a 30 y $40{ }^{\circ} \mathrm{C}$ durante $2 \mathrm{~h}$ empleando las siguientes enzimas: 1) Poligalacturonasa (Po) proveniente del Aspergillus niger (Sigma, E 17389, presentación en polvo) y 2) Pectinex Ultra Clear ( $\mathrm{Pe}$ ) proveniente del Aspergillus aculeatus (Sigma, E 6287, liquida). Se preparó previamente una solución enzimática al $1 \%$ y se, dosifico $0,0.5,1,1.5 \mathrm{ml}$ de la solución enzimática preparada a cada vaso conteniendo $100 \mathrm{~g}$ de sauco triturado. La extracción se realizó al pH natural de las bayas de sauco.

Tratamiento con ultrasonido (US) se realizó con un procesador ultrasónico UP $100 \mathrm{H}$, fabricado por Hielscher Ultrasonics $\mathrm{GmbH}$, con las siguientes características: potencia 100 Watts, frecuencia $30 \mathrm{KHz}$, sonotrodo del tipo MS/ titanio $7 \mathrm{~mm}$ de diámetro, longitud $80 \mathrm{~mm}$, amplitud de potencia regulable de 20 a $100 \%$, intensidad de pulso de 0.2 a 1, se evaluaron potencias de 20, 60 y $100 \%$ de amplitud, pulsos de $0.2,0.6$ y 1.0, por un tiempo de tres minutos en cada caso. Se sumergió el sonotrodo a una profundidad de $1.5 \mathrm{~cm}$, equivalente a la mitad de la altura del volumen ocupado por $100 \mathrm{~g}$ de sauco triturado en un vaso de $250 \mathrm{ml}$ y que fue de $3.2 \mathrm{~cm}$. Durante la aplicación se agito el vaso y se mantuvo constante la temperatura alrededor de $20^{\circ} \mathrm{C}$.

La extracción de antioxidantes empleando microondas (MO) se efectuó en un microondas de marca Interwabe grill de $2455 \mathrm{GHz}$, se colocó el vaso de $250 \mathrm{ml}$ con el sauco triturado en el plato del microondas y se aplicó una exposición por $30 \mathrm{~s}$ (1 ciclo), luego la muestra se enfrió a temperatura ambiente, si la muestra se expone a mas ciclos, la muestra se coloca nuevamente en el microondas, terminada la exposición se retira la muestra ,se repite de esa manera según el número total de ciclos establecidos; en el tratamiento, en cada ciclo la temperatura se eleva de $18-20{ }^{\circ} \mathrm{C}$ hasta $65-67{ }^{\circ} \mathrm{C}$, se enfría la muestra después de cada ciclo aplicado. Se realizó extracciones con 1, 2,4, 6 y 8 ciclos.

Finalmente se combinaron los siguientes tratamientos de extracción: a) Extracción con enzimas seguida de la aplicación de ultrasonido y b) Extracción con enzimas seguida por el empleo de microondas. Después de cada tratamiento de extracción evaluado, la muestra se prensa y se efectúo los análisis de antocianinas, fenoles totales y capacidad antioxidante en el zumo obtenido después del prensado y centrifugado.

\section{Procedimiento de elaboración de la bebida}

Con el mejor tratamiento de extracción se elaboró una bebida de sauco, para tal fin el zumo de sauco obtenido se diluye en una relación 1:2 (uno de jugo o extracto de sauco y dos de agua) adoptada de acuerdo a experimentos previos realizados que determinan buenas características físico químicas y sensoriales, se pasteurizo por $10 \mathrm{~min}$ a $85-88^{\circ} \mathrm{C}$, se añadió $200 \mathrm{ppm}$ de sorbato de potasio y se regulo los ${ }^{\circ}$ $\mathrm{Bx}$ a $12^{\circ}$ con sacarosa, el envasado se realizó en caliente en botellas de vidrio de $298 \mathrm{ml}$ de capacidad y se mantuvo en refrigeración $\left(6 \mathrm{a} 8^{\circ} \mathrm{C}\right)$ protegido de la luz. Durante tres meses la bebida fue evaluada determinando del nivel de degradación del contenido de antocianinas y variación del contenido de fenoles totales y capacidad antioxidante. 
En cada caso las variables a evaluar en los zumos extraídos fueron: contenido de antocianinas, fenoles totales, capacidad antioxidante y rendimiento volumétrico de los zumos obtenidos al aplicar los diversos tratamientos de extracción., para tal fin se efectuaron los siguientes análisis, previa centrifugación del zumo: Antocianinas por el método diferencial de pH (Giusti y Wrolstad 2003; Lee et al., 2005), el contenido de antocianinas se expresó como $\mathrm{mg} / \mathrm{l}$ de cianidina-3-glucosido, los fenoles totales se determinaron por el método de Folin Ciocalteau, la Capacidad antioxidante $(\mathrm{CA})$ por los métodos Cuprac (reducción del ion cúprico) y ABTS (captura del radical catión del ácido 2,2'-azino-bis-(3-etill benzotiazolin-6-sulfonico), los resultados del contenido de fenoles totales y capacidad antioxidante se expresaron como equivalentes a la acción del Trolox, según lo descrito por Guclu et al., 2006. Se empleó 6-hidroxi-2, 5, 7,8 tetramethilchroman -2-acido carboxílico (Trolox, TR) como estándar en la elaboración de las rectas de calibración.

Se realizaron también los análisis indicados en el zumo obtenido de las bayas trituradas sin aplicación de tratamiento de extracción denominado zumo control (ST) y cuyos valores se toman como referencia asignándoles un valor del $100 \%$. El volumen extraído en los diversos tratamientos aplicados se expresó como rendimiento de extracción, el cual se determinó dividiendo el volumen de sauco obtenido en el prensado entre el volumen del zumo control obtenido de la muestra sin tratamiento (ST). Los resultados de las características antioxidantes obtenidos en los diversos tratamientos fueron comparados con los obtenidos en el zumo control. Los análisis se realizaron previa centrifugación del zumo.

Los análisis físico-químicos efectuados en el zumo control y en la bebida elaborada fueron pH según el método de la AOAC (1990) empleando un potenciómetro Jenway 3510, solidos solubles empleando un refractómetro ABBE AR 12, densidad empleando el método del picnómetro y acidez total por titulación con $\mathrm{NaOH} 0.1 \mathrm{~N}$ hasta un $\mathrm{pH}$ de 8.2 , el porcentaje de acidez se expresó como acido tartárico

\section{Análisis estadístico de los datos}

Las determinaciones de $\mathrm{pH}$ y acidez se realizaron por duplicado y los diversos tratamientos de extracción por triplicado determinándose el valor medio. Se efectuó el análisis de varianza y pruebas de comparación de medias de tratamientos con un control, prueba de Dunnett. Se utilizó programas informáticos Microsoft Excel y un paquete estadístico SPSS v 18.

\section{RESULTADOS Y DISCUSION}

Se presentan los resultados y se discuten en varias subsecciones: Características físicas químicas y antioxidantes del zumo control, extracción de antioxidantes por: maceración, extracción de antioxidantes con enzimas, con ultrasonido, extracción de antioxidantes empleando microondas, extracción de antioxidantes empleando tratamientos combinados, rendimientos de extracción de jugo, y bebida antioxidante de bayas de sauco

\section{Características físico químicas y antioxidantes del zumo control}

Las características físico químicas del zumo control de las bayas de sauco fueron: $\mathrm{pH}$ : 3.5-3.6, ${ }^{\circ} \mathrm{Bx}$ : 9.0 9.5, densidad: 1.039, Acidez (g de ácido tartárico/l): 25.5-27.5. El valor de $\mathrm{pH}$ del jugo es favorable a la estabilidad de las antocianinas y está en relación a su alta acidez (López et al., 2007). Valores de pH superiores provocan un cambio en la coloración, por otro lado el bajo valor de pH permite la aplicación de tratamientos térmicos de pasteurización en la elaboración de bebidas.

Las características antioxidantes del zumo control obtenido de bayas trituradas fueron: Antocianinas: 438 mg/l, Fenoles totales: 47948, CA Cuprac: 31835, CA ABTS: $19945 \mu \mathrm{M} \mu \mathrm{mol}$ TR/l, siendo el volumen extraído de jugo $74 \mathrm{ml}$. A dichos valores se les asigno un valor del $100 \%$ y permitió comparar el nivel de eficacia de los tratamientos de extracción evaluados.

\section{Extracción de antioxidantes por maceración}

El tratamiento aplicado de maceración permitió obtener zumos con diversas características antioxidantes según se observa en la Tabla 1. Se observa que las características antioxidantes mejoran con el tiempo de exposición y con la temperatura, siendo favorable $70^{\circ} \mathrm{C}$; a $80^{\circ} \mathrm{C}$ estas decrecieron, se alcanzó valores máximos en antocianinas en $20 \mathrm{~min}$ a $70{ }^{\circ} \mathrm{C}$, a 20 min la extracción se incrementó a $165 \%$ (antocianinas), $130 \%$ (fenoles totales), $121 \%$ (CA Cuprac) y $114 \%$ (ABTS) con respecto al zumo control (sin tratamiento),lo cual se explica por el incremento de la solubilidad de los componentes polifenolicos presentes en las bayas. Galic et al. (2009) describieron incrementos de antocianinas y de poli fenoles en bayas de sauco en los procesos de extracción: que implico etapas de desintegración de las bayas, 
blanqueo, prensado, siendo el blanqueo efectuado a $70{ }^{\circ} \mathrm{C}$ por 10 minutos, en el presente trabajo se efectuó de manera previa una desintegración de las bayas de sauco en el procesador de alimentos y luego un proceso de maceración a las condiciones señaladas.

Tabla 1: Características antioxidantes de zumos obtenidos por maceración

\begin{tabular}{|c|c|c|c|c|c|c|c|c|}
\hline \multirow[t]{2}{*}{ Condición } & \multicolumn{2}{|c|}{ Antocianinas } & \multicolumn{2}{|c|}{ Fenoles totales } & \multicolumn{2}{|c|}{ CA Cuprac } & \multicolumn{2}{|c|}{ CA ABTS } \\
\hline & $\mathrm{mg} / \mathrm{l}$ & $\%$ & $\mu \mathrm{mol}$ TR/I & $\%$ & $\mu \mathrm{mol}$ TR/l & $\%$ & $\mu \mathrm{mol}$ TR/I & $\%$ \\
\hline \multicolumn{9}{|c|}{ Zumo control } \\
\hline---- & 438 & 100 & 47948 & 100 & 31835 & 100 & 19945 & 100 \\
\hline $\mathrm{T}\left({ }^{\circ} \mathrm{C}\right)$ & \multicolumn{8}{|c|}{10 minutos } \\
\hline 30 & 564 & 129 & 57323 & 120 & 31275 & 98 & 18060 & 91 \\
\hline 50 & 631 & 144 & 58923 & 123 & 31760 & 100 & 18629 & 93 \\
\hline 70 & 675 & 154 & 59723 & 125 & 34002 & 107 & 21459 & 108 \\
\hline$t(\min )$ & \multicolumn{8}{|c|}{$70^{\circ} \mathrm{C}$} \\
\hline 5 & 534 & 122 & 50348 & 105 & 30706 & 96 & 17205 & 86 \\
\hline 10 & 675 & 154 & 56960 & 119 & 37609 & 118 & 19828 & 99 \\
\hline 20 & 721 & 165 & 62210 & 130 & 38366 & 121 & 22745 & 114 \\
\hline 30 & 711 & 163 & 64685 & 135 & 38836 & 122 & 23158 & 116 \\
\hline$t(\min )$ & \multicolumn{8}{|c|}{$80^{\circ} \mathrm{C}$} \\
\hline 5 & 556 & 127 & 50198 & 105 & 30654 & 96 & 18295 & 92 \\
\hline 10 & 558 & 127 & 48210 & 101 & 29563 & 93 & 17146 & 86 \\
\hline 20 & 513 & 117 & 49448 & 103 & 28972 & 91 & 16625 & 83 \\
\hline 30 & 516 & 118 & 49223 & 103 & 30290 & 95 & 17559 & 88 \\
\hline
\end{tabular}

La temperatura de $80{ }^{\circ} \mathrm{C}$ asociada al tiempo de extracción afecto la estabilidad de las características antioxidantes de los zumos de sauco obtenidos; en el caso de las antocianinas se degradaron por efecto de la temperatura, algunos autores mencionan que a temperaturas superiores a $40{ }^{\circ} \mathrm{C}$ las antocianinas sufren degradación (Giusti y Wrolstad, 2001). Por lo expuesto las condiciones favorables de extracción de antocianinas en el tratamiento por maceración fueron de $70^{\circ} \mathrm{C}$ por $20 \mathrm{~min}$

\section{Extracción de antioxidantes con enzimas}

En cuanto al tratamiento enzimático (Tabla 2), las características antioxidantes de los zumos obtenidos se incrementaron con la temperatura de incubación de 30 a $40^{\circ} \mathrm{C}$ con ambos tipos de enzimas empleadas; con una dosificación de $1 \mathrm{ml}$ de solución enzimática, las antocianinas aumentaron de $108 \%$ a $134 \%$ con Petinex ultra Clear (Pe), y con la Poligalacturonasa (Po) de 112 a $134 \%$; los fenoles totales y la CA Cuprac y ABTS también mostro incrementos. Este incremento esta en relación con la temperatura óptima de actividad de las enzimas que en este caso fue de $40^{\circ} \mathrm{C}$ por 2 horas. Al igual que el trabajo desarrollado por Buchert et al. (2005), el tipo de baya y enzima también influye en los niveles de extracción. Los resultados encontrados son sin embargo menores a los obtenidos por maceración a $70{ }^{\circ} \mathrm{C}$. La aplicación de enzimas en la recuperación de antocianinas favorece los rendimientos de extracción en orujos de uva (Maier et al., 2007).

Por lo expuesto el tratamiento enzimático fue favorable a la temperatura de $40{ }^{\circ} \mathrm{C}, 2 \mathrm{~h}$ de incubación y con una dosificación de $1 \mathrm{ml}$ de solución enzimática para ambos tipos de enzimas sin embargo los resultados fueron menores a los obtenidos por maceración.

\section{Extracción de antioxidantes con ultrasonido}

La Tabla 3 muestra los resultados obtenidos tras el tratamiento con ultrasonido (US), el contenido de antocianinas se incrementó a $110 \%$ y fenoles totales a $117 \%$ con una intensidad de pulso de 1, amplitud de 100 y tres min de tratamiento. El incremento no parece ser favorable cuando aumenta el tiempo de extracción de 3 a 5 min, pero si cuando se aumenta la intensidad de pulso; los incrementos en capacidad antioxidante no son relevantes, las muestras se mantuvieron en $20^{\circ} \mathrm{C}$ en todos los casos

La aplicación de ultrasonido resulta una técnica eficaz para la extracción de antocianinas de bayas usando bajas temperaturas y un tiempo razonable (Celli et al., 2015). La ventaja de la aplicación de ultrasonido es la reducción de tiempo en la extracción frente a los métodos convencionales observados (Carrera et al., 2012). Los niveles de extracción de antioxidantes fueron sin embargo menores a los obtenidos por maceración a $70{ }^{\circ} \mathrm{C}$ y $20 \mathrm{~min}$. Las mejores condiciones de extracción con ultrasonido fueron al aplicar una amplitud de 100, 3 min de tratamiento e intensidad de pulso de 1 . 
Tabla 2: Tratamiento enzimático en la extracción de antioxidantes en bayas de sauco

\begin{tabular}{|c|c|c|c|c|c|c|c|c|}
\hline \multirow{2}{*}{$\begin{array}{c}\text { Dosificación } \\
(\mathrm{ml})\end{array}$} & \multicolumn{2}{|c|}{ Antocianinas } & \multicolumn{2}{|c|}{ Fenoles totales } & \multicolumn{2}{|c|}{ CA Cuprac } & \multicolumn{2}{|c|}{ CA ABTS } \\
\hline & $\mathrm{mg} / \mathrm{l}$ & $\%$ & $\mu \mathrm{mol} \mathrm{TR} / \mathrm{I}$ & $\%$ & $\mu \mathrm{mol}$ TR/I & $\%$ & $\mu \mathrm{mol}$ TR/I & $\%$ \\
\hline \multicolumn{9}{|c|}{ Zumo control } \\
\hline--- & 438 & 100 & 47948 & 100 & 31835 & 100 & 19945 & 100 \\
\hline \multicolumn{9}{|c|}{$\mathrm{Pe}-30^{\circ} \mathrm{C}$} \\
\hline 0 & 440 & 101 & 48135 & 100 & 31358 & 99 & 18413 & 92 \\
\hline 0.5 & 466 & 106 & 48773 & 102 & 34040 & 107 & 19062 & 96 \\
\hline 1.0 & 473 & 108 & 49785 & 104 & 35677 & 112 & 20447 & 103 \\
\hline 1.5 & 441 & 101 & 48548 & 101 & 31858 & 100 & 19179 & 96 \\
\hline \multicolumn{9}{|c|}{$\mathrm{Pe}-40^{\circ} \mathrm{C}$} \\
\hline 0 & 514 & 118 & 52029 & 109 & 34177 & 107 & 19651 & 99 \\
\hline 0.5 & 566 & 129 & 53198 & 111 & 35040 & 110 & 19563 & 98 \\
\hline 1 & 586 & 134 & 53554 & 112 & 35904 & 113 & 21301 & 107 \\
\hline 1.5 & 532 & 122 & 54557 & 114 & 35199 & 111 & 20633 & 103 \\
\hline 2 & 536 & 123 & 55560 & 116 & 34495 & 108 & 22215 & 111 \\
\hline \multicolumn{9}{|c|}{$\mathrm{Po}-30^{\circ} \mathrm{C}$} \\
\hline 0 & 455 & 104 & 50316 & 105 & 31449 & 99 & 20712 & 104 \\
\hline 0.5 & 485 & 111 & 50935 & 106 & 33245 & 104 & 19690 & 99 \\
\hline 1 & 488 & 112 & 50654 & 106 & 31881 & 100 & 20005 & 100 \\
\hline 1.5 & 491 & 112 & 47441 & 99 & 33131 & 104 & 19474 & 98 \\
\hline \multicolumn{9}{|c|}{$\mathrm{Po}-40^{\circ} \mathrm{C}$} \\
\hline 0 & 528 & 121 & 50816 & 106 & 31835 & 100 & 19003 & 95 \\
\hline 0.5 & 543 & 124 & 52373 & 109 & 34472 & 108 & 21753 & 109 \\
\hline 1 & 585 & 134 & 54023 & 113 & 34654 & 109 & 22775 & 114 \\
\hline 1.5 & 582 & 133 & 55213 & 115 & 34393 & 108 & 21709 & 109 \\
\hline 2 & 485 & 111 & 54185 & 113 & 33540 & 105 & 21743 & 109 \\
\hline
\end{tabular}

Tabla 3: Características antioxidantes de zumos de sauco obtenidos por extracción asistida por ultrasonido

\begin{tabular}{|c|c|c|c|c|c|c|c|c|}
\hline \multirow[t]{2}{*}{ Pulsos } & \multicolumn{2}{|c|}{ Antocianinas } & \multicolumn{2}{|c|}{ Fenoles totales } & \multicolumn{2}{|c|}{ CA Cuprac } & \multicolumn{2}{|c|}{ CA ABTS } \\
\hline & $\mathrm{mg} / \mathrm{l}$ & $\%$ & $\mu \mathrm{mol} \mathrm{TR} / \mathrm{l}$ & $\%$ & $\mu \mathrm{mol} \mathrm{TR} / \mathrm{l}$ & $\%$ & $\mu \mathrm{mol} \mathrm{TR} / \mathrm{l}$ & $\%$ \\
\hline \multicolumn{9}{|c|}{ Zumo control } \\
\hline$-\cdots$ & 438 & 100 & 47948 & 100 & 31835 & 100 & 19945 & 100 \\
\hline \multicolumn{9}{|c|}{ Amplitud 20, 3 min } \\
\hline 0.2 & 456 & 104 & 51473 & 107 & 32381 & 102 & 19813 & 99 \\
\hline 0.6 & 466 & 106 & 52166 & 109 & 32472 & 102 & 19533 & 98 \\
\hline 1 & 458 & 105 & 52110 & 109 & 32529 & 102 & 19739 & 99 \\
\hline \multicolumn{9}{|c|}{ Amplitud 60, 3 min } \\
\hline 0.2 & 465 & 106 & 53048 & 111 & 32256 & 101 & 19150 & 96 \\
\hline 0.6 & 463 & 106 & 53329 & 111 & 33438 & 105 & 19297 & 97 \\
\hline 1 & 467 & 107 & 54810 & 114 & 32620 & 102 & 19386 & 97 \\
\hline \multicolumn{9}{|c|}{ Amplitud 100, 3 min } \\
\hline 0.2 & 459 & 105 & 52204 & 109 & 31688 & 100 & 19906 & 100 \\
\hline 0.6 & 470 & 107 & 53265 & 111 & 33034 & 104 & 20108 & 101 \\
\hline 1 & 481 & 110 & 56035 & 117 & 34949 & 110 & 19504 & 98 \\
\hline \multicolumn{9}{|c|}{ Amplitud 100, 5 min } \\
\hline 0.2 & 461 & 105 & 50447 & 105 & 31957 & 100 & 20005 & 100 \\
\hline 0.6 & 473 & 108 & 51095 & 107 & 31676 & 100 & 20270 & 102 \\
\hline 1 & 453 & 104 & 51041 & 106 & 32229 & 101 & 19622 & 98 \\
\hline
\end{tabular}

\section{Extracción de antioxidantes empleando Microondas}

Los rendimientos de extracción empleando microondas (MO), Tabla 4 fueron favorables a partir de la aplicación de cuatro ciclos, siendo cercanos a los obtenidos por maceración, tienen la ventaja que los tiempos de extracción son reducidos, el tiempo efectivo de extracción en cuatro ciclos aplicados suman 2 min. Diversos estudios demuestran que la extracción con microondas favorecen el rendimiento de extracción de polifenoles y la reducción del tiempo de tratamiento en bayas chinas y en cascaras de uva (Duan et al., 2015, Yu H. et al., 2013).

Cabe mencionar la forma diferente de extracción de estas sustancias por los diferentes métodos aplicados, en la maceración básicamente fue el efecto térmico y el tiempo de exposición; el tratamiento enzimático por su lado actúa sobre la modificación de la estructura de la pared celular, siendo las enzimas empleadas del tipo pectinoesterasas que aumentaron los rendimientos de extracción y también la temperatura de incubación; en el tratamiento aplicado por ultrasonido básicamente su acción fue disruptiva por las fuerzas 
de cavitación generadas, reduciendo el tamaño de partícula para favorecer la difusión de sustancias extraíbles al medio líquido, los tiempos de aplicación fueron cortos y la temperatura se mantuvo sin variación alrededor de $20^{\circ} \mathrm{C}$. El tratamiento de microondas genera un efecto térmico al aumentar la temperatura en un tiempo breve en cada ciclo por efecto de la vibración de las moléculas de agua por efecto de la energía electromagnética generada en el microondas que favorecen la difusión de componentes antioxidantes al medio líquido. Por lo expuesto, una aplicación de cuatro ciclos de exposición con microondas de 30 seg cada uno genera una mayor extracción de antocianinas.

Tabla 4: Extracción de antioxidantes con microondas

\begin{tabular}{|c|c|c|c|c|c|c|c|c|}
\hline \multirow{2}{*}{ Ciclos } & \multicolumn{2}{|c|}{ Antocianinas } & \multicolumn{2}{c|}{ Fenoles totales } & \multicolumn{2}{c|}{ CA Cuprac } & \multicolumn{2}{c|}{ CA ABTS } \\
\cline { 2 - 8 } & $\mathrm{mg} / \mathrm{l}$ & $\%$ & $\mu \mathrm{mol}$ TR/l & $\%$ & $\mu \mathrm{mol}$ TR/I & $\%$ & $\mu \mathrm{mol}$ TR/I & $\%$ \\
\hline \multicolumn{8}{|c|}{ Zumo control } \\
\hline--- & 438 & 100 & 47948 & 100 & 31835 & 100 & 19945 & 100 \\
\hline \multicolumn{8}{|c|}{ Extracción con Microondas } \\
\hline 1 & 506 & 116 & 55710 & 116 & 33563 & 105 & 21066 & 106 \\
\hline 2 & 534 & 122 & 55560 & 116 & 34608 & 109 & 21301 & 107 \\
\hline 4 & 696 & 159 & 56760 & 118 & 35495 & 111 & 22745 & 114 \\
\hline 6 & 667 & 152 & 56910 & 119 & 37222 & 117 & 23158 & 116 \\
\hline 8 & 664 & 152 & 57060 & 119 & 38609 & 121 & 23158 & 116 \\
\hline
\end{tabular}

\section{Extracción de antioxidantes empleando tratamientos combinados}

La combinación Enzima-Ultrasonido (Tabla 5) mostro un efecto favorable en la extracción de antioxidantes comparándolas a la sola aplicación de ultrasonido, se observa sin embargo que el tratamiento enzimático por si solo alcanzó mejores niveles. Resultados hallados por Tchavo et al., 2015, demostraron el efecto favorable de dicha combinación aplicada en moras. El tratamiento aplicado fue: dosificación de $1 \mathrm{ml}$ de solución enzimática, temperatura de $40^{\circ} \mathrm{C}, 2 \mathrm{~h}$ de incubación, el tratamiento con ultrasonido fue de amplitud 100, 3 min de exposición y pulsación de 1 en cada caso.

Tabla 5: Extracción de antioxidantes empleando Enzimas y Ultrasonido

\begin{tabular}{|c|c|c|c|c|c|c|c|c|c|}
\hline \multirow{3}{*}{ Tratamiento } & \multicolumn{2}{|c|}{ Antocianinas } & \multicolumn{2}{c|}{ Fenoles totales } & \multicolumn{2}{c|}{ CA Cuprac } & \multicolumn{2}{c|}{ CA ABTS } \\
\cline { 2 - 9 } & $\mathrm{mg} / \mathrm{l}$ & $\%$ & $\mu \mathrm{mol}$ TR/I & $\%$ & $\mu \mathrm{mol}$ TR/I & $\%$ & $\mu \mathrm{mol}$ TR/I & $\%$ \\
\cline { 2 - 9 } & \multicolumn{7}{|c|}{ \% } & \multicolumn{7}{c|}{ Zumo control } \\
\hline---- & 438 & 100 & 47948 & 100 & 31835 & 100 & 19945 & 100 \\
\hline $\mathrm{Pe}+$ US & 582 & 133 & 53610 & 112 & 33517 & 105 & 20181 & 101 \\
\hline $\mathrm{Pe}$ & 586 & 134 & 53554 & 112 & 35904 & 113 & 21301 & 107 \\
\hline $\mathrm{Po}+$ US & 575 & 131 & 52729 & 110 & 33639 & 106 & 20515 & 103 \\
\hline Po & 585 & 134 & 54023 & 113 & 34654 & 109 & 22775 & 114 \\
\hline US & 481 & 110 & 56035 & 117 & 34949 & 110 & 19504 & 98 \\
\hline
\end{tabular}

La combinación Enzima- Microondas (Tabla 6) mostró un efecto favorable en la extracción de antocianinas al combinar la enzima Petinex ultra Clear $(\mathrm{Pe})$ con el tratamiento por microondas; no se observó incremento al combinar la Poligalacturonasa (Po) con microondas con respecto a la sola extracción con microondas. El tratamiento aplicado correspondió a la aplicación de 4 ciclos cuando se emplea microondas, y dosificación de $1 \mathrm{ml}$ de la solución enzimática respectiva en cada caso, temperatura de incubación de $40{ }^{\circ} \mathrm{C}$ y $2 \mathrm{~h}$ de tratamiento en baño maría.

Tabla 6:.Extracción de antioxidantes empleando enzimas en combinación con microondas

\begin{tabular}{|c|c|c|c|c|c|c|c|c|}
\hline \multirow{2}{*}{ Condición } & \multicolumn{2}{|c|}{ Antocianinas } & \multicolumn{2}{c|}{ Fenoles totales } & \multicolumn{2}{c|}{ CA Cuprac } & \multicolumn{2}{c|}{ CA ABTS } \\
\cline { 2 - 9 } & $\mathrm{mg} / \mathrm{l}$ & $\%$ & $\mu \mathrm{mol}$ TR/I & $\%$ & $\mu \mathrm{mol}$ TR/l & $\%$ & $\mu \mathrm{mol}$ TR/I & $\%$ \\
\hline \multicolumn{8}{|c|}{ Zumo control } \\
\hline----- & 438 & 100 & 47948 & 100 & 31835 & 100 & 19945 & 100 \\
\hline $\mathrm{Pe}+\mathrm{MO}$ & 710 & 162 & 56929 & 119 & 35654 & 112 & 22343 & 112 \\
\hline $\mathrm{Pe}$ & 586 & 134 & 53554 & 112 & 35904 & 113 & 21301 & 107 \\
\hline $\mathrm{Po}+\mathrm{MO}$ & 696 & 159 & 54585 & 114 & 34548 & 109 & 21694 & 109 \\
\hline $\mathrm{Po}$ & 585 & 134 & 54023 & 113 & 34654 & 109 & 22775 & 114 \\
\hline $\mathrm{MO}$ & 696 & 159 & 56760 & 118 & 35495 & 111 & 22745 & 114 \\
\hline
\end{tabular}

Las tablas 1 a 6 muestran la comparación de porcentajes de eficacia en la extracción de: Antocianinas, fenoles totales y características de la capacidad antioxidante respecto al zumo control, en algunos casos son menores al $100 \%$ sobre todo a nivel de ABTS, resultados que están en relación con el nivel de intensidad del tratamiento aplicado y las características del método ABTS en identificar sustancias antioxidantes generadas en el proceso de extracción 
La Tabla 7 muestra el rendimiento de extracción en volumen de zumo tras la aplicación de los diversos tratamientos con respecto al zumo control. Un mayor rendimiento de extracción en volumen se obtuvo tras el tratamiento enzimático (dosificación de $1 \mathrm{ml}$ de solución enzimática) frente a otros como maceración, ultrasonido (amplitud 100, 3 min de exposición, pulsación de 1) y microondas (4 ciclos). Las enzimas empleadas alteran la estructura de la pared celular y mejoran el rendimiento de extracción del jugo, como consecuencia se liberan componentes fenólicos al jugo. Se desprende que un tratamiento enzimático previo al de maceración podría favorecer el incremento de la extracción en volumen.

Tabla 7: Rendimiento de extraccion de zumo

\begin{tabular}{|l|c|}
\hline \multicolumn{1}{|c|}{ Método de Extracción } & Rendimiento (\%) \\
\hline Zumo control & 100 \\
\hline Maceración $\left(70{ }^{\circ} \mathrm{C}\right)$ & 104 \\
\hline Petinex ultra Clear $(\mathrm{Pe})$ & 114 \\
\hline Poligalacturonasa $(\mathrm{Po})$ & 113 \\
\hline $\mathrm{Pe}+\mathrm{US}$ & 114 \\
\hline $\mathrm{Po}+\mathrm{US}$ & 112 \\
\hline Ultrasonido (US) & 105 \\
\hline Microondas $(\mathrm{MO})$ & 104 \\
\hline $\mathrm{Pe}+\mathrm{MO}$ & 114 \\
\hline $\mathrm{Po}+\mathrm{MO}$ & 111 \\
\hline
\end{tabular}

Los diferentes resultados obtenidos en cada tratamiento aplicado fueron sometidos al análisis estadístico, se realizó el análisis de varianza. En todos los casos se encontró que el valor del cociente $F_{0}$ fue mayor al comparar con un punto porcentual de la tabla de distribución hallado en tablas ( $\left.\mathrm{F}_{0}>\mathrm{F}_{\mathrm{Tabla}}\right)$ y que denotan diferencias en las medias de los tratamientos, excepto para los resultados ABTS en el tratamiento con Ultrasonido, Al aplicar la prueba de Dunnett para la comparación de medias de tratamientos con el zumo control se precisó estas diferencias entre los diversos métodos evaluados.

La Tabla 8, muestra en resumen los resultados favorables de extracción en antocianinas y sus correspondientes niveles de polifenoles totales, Capacidad antioxidante: Cuprac y ABTS, para los diversos métodos de extracción aplicados. Se muestra la diferencia entre la medias de los tratamientos efectuados respecto a la diferencia critica hallada por la prueba de Dunnett con un valor de significancia de 0.05 , grado de libertad de 9 en la fuente de variación (a-1) y un grado de libertad del error (f) de 20.

Tabla 8: Niveles de antioxidantes en diversos métodos de extracción

\begin{tabular}{|l|c|c|c|c|c|c|c|c|}
\hline \multirow{2}{*}{ Método de Extracción } & \multicolumn{2}{|c|}{ Antocianinas } & \multicolumn{2}{c|}{ Fenoles totales } & \multicolumn{2}{c|}{ CA Cuprac } & \multicolumn{2}{c|}{ CA ABTS } \\
\cline { 2 - 9 } & $\%$ & $\begin{array}{c}\text { Dif>Prueba } \\
\text { Dunnett }\end{array}$ & $\%$ & $\begin{array}{c}\text { Dif>Prueba } \\
\text { Dunnett }\end{array}$ & $\%$ & $\begin{array}{c}\text { Dif }>\text { Prueba } \\
\text { Dunnett }\end{array}$ & $\begin{array}{c}\% \\
\text { Dif }>\text { Prueba } \\
\text { Dunnett }\end{array}$ \\
\hline Zumo control & 100 & --- & 100 & --- & 100 & --- & 100 & --- \\
\hline Maceración $\left(70^{\circ} \mathrm{C}\right)$ & 165 & $283>21$ & 130 & $14262>783$ & 121 & $6531>1024$ & 114 & $2800>1229$ \\
\hline Petinex ultra Clear $(\mathrm{Pe})$ & 134 & $148>21$ & 112 & $5606>783$ & 113 & $4069>1024$ & 107 & $1356>1229$ \\
\hline Poligalacturonasa (Po) & 134 & $147>21$ & 113 & $6075>783$ & 109 & $2819>1024$ & 114 & $2830>1229$ \\
\hline Ultrasonido (US) & 110 & $43>21$ & 117 & $8087>783$ & 110 & $3114>1024$ & 98 & $-441<1229$ \\
\hline Pe + US & 133 & $144>21$ & 112 & $5662>783$ & 105 & $1682>1024$ & 101 & $236<1229$ \\
\hline Po + US & 131 & $137>21$ & 110 & $4781>783$ & 106 & $1804>1024$ & 103 & $570<1229$ \\
\hline Microondas (MO) & 159 & $258>21$ & 118 & $8812>783$ & 111 & $3660>1024$ & 114 & $2800>1229$ \\
\hline Pe + MO & 162 & $272>21$ & 119 & $8981>783$ & 112 & $3819>1024$ & 112 & $2398>1229$ \\
\hline Po + MO & 159 & $258>21$ & 114 & $6637>783$ & 109 & $2713>1024$ & 109 & $1749>1229$ \\
\hline
\end{tabular}

Se observa que los resultados de los diversos métodos de extracción aplicados son mayores al zumo control por lo que es de resaltar dicho efecto, la extracción por maceración mostro los mejores resultados de extracción en antocianinas seguida por el tratamiento con microondas, esta última incremento el rendimiento de extracción de antocianinas al combinarse con la enzima Pectinex ultra clear. En la aplicación de ultrasonido y el uso de enzimas - ultrasonido, la diferencia de medias de los resultados ABTS son menores a la diferencia crítica hallada con la prueba de Dunnett, en dichas condiciones no fueron significativamente 
diferentes con respecto al control. El porcentaje de extracción al aplicar ultrasonido alcanzo un valor del 98 $\%$ respecto del control (100\%); cuando se combinan los dos tipos de enzimas con ultrasonido el incremento es mínimo respecto al control. En todos los demás casos la diferencia de medias de los tratamiento aplicados son mayores a la diferencia critica de Dunnett, y que permitió diferenciar los niveles de extracción y capacidad antioxidante por cada método evaluado y determinar estadísticamente un orden de eficacia. Cabe resaltar el hecho de la relación que se da en la aplicación de la prueba de Dunnett de comparación de medias con la comparación en porcentajes en valor absoluto respecto al zumo control que también señala la eficacidad del método empleado.

\section{Bebida antioxidante de bayas de sauco}

Las bebidas antioxidantes fueron elaboradas con el zumo de sauco obtenido por maceración a $70{ }^{\circ} \mathrm{C}$ durante $20 \mathrm{~min}$, diluidas, pasteurizadas, envasadas y mantenidas a $6-8^{\circ} \mathrm{C}$ en refrigeración como se señaló en los procedimientos. Las características físico químicas de la bebida elaborada de sauco fueron de: $\mathrm{pH}$ 3.6, ${ }^{\circ} \mathrm{Bx} 12$ y acidez ( $\mathrm{g}$ de ácido tartárico/l): 8 . Siendo el zumo natural de las bayas del sauco muy acidas se efectuaron pruebas preliminares sensoriales y físico químicas a varias diluciones, siendo la dilución 1:2(uno de zumo natural y 2 de agua), la que dio mejores resultados. Las características antioxidantes: Contenido de antocianinas, fenoles totales y capacidad antioxidante se determinaron en la bebida después de elaborada y durante 90 días, resultados que se muestran en la Tabla 9.

Tabla 9: Características antioxidantes de la bebida de sauco durante el almacenamiento

\begin{tabular}{|c|c|c|c|c|c|c|c|c|}
\hline \multirow{2}{*}{$\begin{array}{c}\text { Tiempo } \\
\text { (días) }\end{array}$} & \multicolumn{2}{|c|}{ Antocianinas } & \multicolumn{2}{c|}{ Fenoles totales } & \multicolumn{2}{c|}{ CA Cuprac } & \multicolumn{2}{c|}{ CA ABTS } \\
\cline { 2 - 9 } & $\mathrm{mg} / \mathrm{l}$ & $\%$ & $\mu \mathrm{mol}$ TR/I & $\%$ & $\mu \mathrm{mol}$ TR/I & $\%$ & $\mu \mathrm{mol}$ TR/l & $\%$ \\
\hline 0 & 298 & 100 & 22664 & 100 & 14568 & 100 & 10079 & 100 \\
\hline 28 & 271 & 91 & 20795 & 92 & 14130 & 97 & 9637 & 96 \\
\hline 63 & 266 & 89 & 20895 & 92 & 14032 & 96 & 9584 & 95 \\
\hline 90 & 266 & 89 & 19808 & 87 & 14011 & 96 & 9532 & 95 \\
\hline
\end{tabular}

Se observa a los 28 d una degradación de las características antioxidantes de las bebidas de sauco elaboradas, alcanzando $91 \%$ de antocianinas, $92 \%$ en fenoles totales, $97 \%$ CA Cuprac, $96 \%$ CA ABTS con respecto al nivel inicial y que podría deberse a la presencia de oxígeno en el espacio de cabeza del envase que hubiera podido quedar atrapado como consecuencia del envasado en caliente realizado; el oxígeno resulta un factor importante a considerar en la degradación de antocianinas (López, 2007); después de los $28 \mathrm{~d}$ las características permanecen estables, al final de los $90 \mathrm{~d}$ de almacenamiento las bebidas de sauco decrecen en total en $11 \%$ antocianinas, $13 \%$ fenoles totales, $4 \%$ en la CA Cuprac y $5 \%$ en la CA ABTS; en términos generales las características antioxidantes permanecen estables después de los 28 d, manteniendo sus características sensoriales de una bebida agradable en color y sabor

\section{CONCLUSIONES}

De los resultados mostrados, de su análisis y discusión se pueden obtener las siguientes conclusiones sobre los diversos métodos de extracción de antioxidantes aplicados a las bayas del sauco y en la elaboración de la bebida.

1) La extracción convencional de maceración aplicada logro obtener las mejores características antioxidantes considerando la temperatura y tiempos adecuados, la extracción con microondas resulta prometedora y con resultados muy cercanos a los de maceración, siendo su ventaja la aplicación de tiempos reducidos, la aplicación de ultrasonido logro incrementos menores.

2) El uso de enzimas y microondas en combinación mejoro la extracción de antioxidantes según el tipo de enzima empleado; el uso combinado de enzimas - ultrasonido no logro incrementar la extracción de antioxidantes con respecto al solo uso de enzimas.

3) El uso de enzimas permitió obtener los mejores rendimientos de extracción de zumo en volumen.

4) La bebida de sauco elaborada tuvo una buena estabilidad durante el periodo de almacenamiento en refrigeración y mostro buenas características sensoriales de color y sabor. 


\section{REFERENCIAS}

Arrasola, G.; Eraso, I. y A. Alvis, Obtención y Evaluación de la Estabilidad de Antocianinas de Berenjena (Solanum melongena L.) en Bebidas, doi: 10.4067/S0718-07642014000300007., Inf. Tecnol. Vol. 25(3), 43$52(2013)$

Ávila-de Hernández, R y J.Bullón-Torrealba, La concentración de jugos de fruta: Aspectos básicos de los procesos sin y con membrana, http://www.scielo.org.ve/scielo.php? , ISSN 0798-4065, Rev. Fac. Ing. UCV, $.28(3) .65-75(2013)$

Buchert,J.; J.M. Koponen y otros cinco autores, Effect of enzyme-aided pressing on anthocyanin yield and profiles in bilberry and blackcurrant juices, doi: 10.1002/jsfa.2284, J. Sci Food Agric ,85 (15): 2548-2556 (2005)

Carrera, C.; Ruiz-Rodríguez, A.; M. Palma y C. Barroso, Ultrasound assisted extraction of phenolic compounds from grapes, doi:10.1016/j.aca.2011.11.032, Analytica chimica acta, vol 732 (Jun), 100-104 (2012)

Celli, G.; Ghanem, A.; M. Brooks y Su-Ling, Optimization of ultrasound-assisted extraction of anthocyanins from haskap berries (Lonicera caerulea L.) using Response Surface Methodology, doi:10.1016/j.ultsonch.2015.06.014, Ultrason. Sonochem, Vol 27(Nov.), 449-455 (2015)

Coronado, M., S. Vega y León y otros tres autores, Antioxidantes: perspectiva actual para la salud humana. http://www.scielo.cl/cgi-bin/wxis.exe/iah/, ISSN 017-7518, Rev. chil. nutr., 42 (2), 206-212 (2015)

Ćujić, N.; K. Savikin y otros cuatro autores, Optimization of polyphenols extraction from dried chokeberry using maceration as traditional technique, doi:10.1016/j.foodchem.2015.08.008, Food Chem.,194 (Mar), 135-142 (2016)

Damodaran, S.; K. Parkin y O. Fennema, Fennema Química de los Alimentos, 3. Edición, pp 1154, Editorial Acribia, S.A, Zaragosa, España, (2010)

Duan, W.; Jin,S.; G. Zhao y P. Sun, Microwave-assisted extraction of anthocyanin from Chinese bayberry and its effects on anthocyanin stability, doi:http://dx.doi.org/10.1590/1678-457X.673, Food Sci. Technol, Campinas, 35(3): 524-530, (2015)

Galić, A.; V.Dragovik-Uzelac y otros cuatro autores, The Polyphenols Stability, Enzyme Activity and PhysicoChemical Parameters During Producing Wild Elderberry Concentrated Juice, http://www.agr.unizg.hr/smotra/issues.htm, ISSN: 1331-7776, Agric. Conspectus. Sci. 74 (3), 181-186 (2009)

García A.; Periago, Maㅡ J.; Mª L Vidal Guevara y E.Cantos, Evaluación de las propiedades antioxidantes en concentrados de uva y frutas rojas, http://revistas.um.es/analesvet/issue/view/1201, ISSN electrónico: 19891784, An. Vet. (Murcia) 18: 103-111 (2002)

Giusti, M y R, Wrolstad, Acylated anthociyanins from edible sources and their applications in food systems. doi:10.1016/S1369-703X(02)00221, Biochem Eng. J. 14:217-25 (2003)

Giusti MM y RE Wrolstad, Unit F1.2: Anthocyanins. Characterization and measurement with UV-visible spectroscopy. In: Wrolstad, RE, Editor. Current protocols in food analytical chemistry. New York: John Wiley \& Sons. p. F1.2.1-1.2.13 (2001)

Guclu, K.; Altun,M.; Ozyurek, M.;S. Karademir y R. Apak, Antioxidant capacity of fresh, sun and sulphited dried Malatya apricot (Prunus amniaca) assayed by CUPRAC, ABTS/TEAC and Folin methods, doi: 10.1111/j.1365-2621.2006.01347.x, International Journal of Food Science and Technology, 41 (Supplement I) $76-85$ (2006)

Jakobek, L.; Seruga, M.; M. Medvidović-Kosanović y I.Novak, Antioxidant Activity and Polyphenols of Aronia in Comparison to other Berry Species., http://www.agr.unizg.hr/smotra/issues.htm, ISSN: 1331-7776, Agriculturae Conspectus Scientificus, 72 (4), 301-306 (2007)

Lee, J.; R. Durst y R. Wrolstad, Determination of total monomeric anthocyanin pigment content of fruit juices, beverages, natural colorants, and wines by the $\mathrm{pH}$ differential method: collaborative study, http://www.ingentaconnect.com/content/aoac/jaoac, ISSN: 1060-3271, J. of AOAC International, 88(5), 12691278 (2005) 
Liazid A.; R. Guerrero y otros tres autores, Microwave assisted extraction of anthocyanins from grape skins, http://www.journals.elsevier.com/food-chemistry, ISSN: 0308-8146, Food Chemistry: 124 (3), 1238-1243 (2012)

López, T.; Fonseca, X. M.; M. Novy Quadri y M.Bastos Quadri, Antocianinas: uma breve revisão das características estruturais e da estabilidade, http://www2.ufpel.edu.br/faem/agrociencia/todas.htm, ISSN:0104-8996, R. Bras. Agrociência, Pelotas, 13 (3), 291-297 (2007)

Maier, T.; A. Goppert y otros tres autores, Optimization of a process for enzyme-assisted pigment extraction from grape (Vitis vinifera L.) pomace, doi: 10.1007/s00217-007-0720-y, European Food Research and Technology, 227 (1), 267-275. (2007)

Vilaplana, M.,Antioxidantes presentes en los alimentos, http://www.elsevier.es/es-revista-offarm-0212047X, ISSN: 0212-047X, Ámbito farmacéutico Nutrición, 26(10), 11-141 (2007)

Volp, A.C.; Renhe I.R.; K. Barra y P.C, Stringueta, Flavonóides antocianinas: características e propiedades na nutrição e saúde, http://portalrev.enfermagem.bvs.br/index.php?issn=0103-7196\&lang=pt, ISSN: 01037196, Rev. Bras. Nutr. Clin. 23(2):141-9 (2008)

Smith, R.; K. Tran y otros seis autores, Elderberry juice composition and health benefits, in Fruit Juices: Types, Nutritional Composition and Health Benefits, by Katherlyn Elizabeth Elder, Editors, Nova Science Publishers, Cap 1, pp:1-20 ISBN 978-1-63321-135-3 (2014)

Tchavo, W.; Ma, Y.; F. Engmann y H. Zhang, Ultrasound-assisted enzymatic extraction (UAEE) of phytochemical compounds from mulberry (Morus nigra) must and optimization study using response surface methodology, doi: 10.1016/j.indcrop.2014.09.053, Industrial Crops and Products, 63,(January), 214-225 (2015)

Yu, Hai Bo.; Ding, Li Fen.; Wang, Zheng y Li X. Shi, Study on Extraction of Polyphenol from Grape Peel Microwave-Assisted Activity, doi:10.4028/www.scientific.net/AMR.864-867.520, Advanced Materials Research, 864-867, 520-525 (2013) 
